\title{
OPEN Sex-differences in psychosocial sequelae after spontaneous cervical artery dissection
}

\author{
Lukas Mayer-Suess ${ }^{1}{ }^{凶}$, Moritz Geiger ${ }^{1}$, Benjamin Dejakum ${ }^{1}$, Christian Boehme $^{1}$, \\ Lena M. Domig ${ }^{1}$, Silvia Komarek ${ }^{1}$, Thomas Toell ${ }^{1}$, Stefan Kiechl ${ }^{1,2}$ \& Michael Knoflach ${ }^{1,2}$
}

Short- to mid-term functional outcome in spontaneous cervical artery dissection is favorable, but the concomitant psychosocial impact is underreported. We aimed to determine these possible sequelae, with a special focus on sex differences, in our cohort of spontaneous cervical artery dissection subjects. During a standardized prospective in-house follow-up visit we, among other values, evaluated functional outcome (modified Rankin Scale [mRS]), psychosocial measures (return to work-, divorce rate) and health-related quality of life (WHO-QoL-BREF and SF-36-questionnaires). 145 patients participated in the long-term prospective follow-up. Median follow-up time was 6.5 years and excellent functional outcome ( $\mathrm{mRS} \leq 1$ ) was achieved in $89.0 \%$ subjects. $87.6 \%$ returned to work and $17.6 \%$ married patients had a divorce during follow-up. Even though relevant baseline-/discharge characteristics and functional outcome did not differ between the sexes, women were less likely to return to work compared to men $(79.7 \%$ vs. $93.8 \% ; \mathrm{P}=0.010)$ and divorce rate was considerably higher in women $(30.2 \%$ vs. $9.2 \% ; P=0.022)$. Health related quality of life did not differ significantly between the sexes, but women consistently reported lower values. Even though functional outcome is beneficial in most patients, measures to prevent poor psychosocial outcome should be considered in the long-term care of patients with spontaneous cervical artery dissection, especially women.

Functional outcome in spontaneous cervical artery dissection (sCeAD) is considered favorable on a short- and medium term ${ }^{1-4}$. As sCeAD is one of the main reasons for ischemic stroke at a young age, it is not surprising that subjects with all-cause cerebral ischemia before the age of 55 share these generally favorable neurological and functional outcomes ${ }^{5,6}$. Still, psychosocial outcome parameters, such as return to work, depression and anxiety proportions are impaired in all-cause young ischemic stroke survivors ${ }^{7-14}$. Interestingly, even though these data exist in all-cause young ischemic stroke patients, psychosocial outcomes have mostly been neglected in $\mathrm{sCeAD}^{1-4}$. The little available data suggest that a sCeAD diagnosis may have profound effect on the psychological state of patients but outcome analyses to date have focused on isolated metrics, such as functional parameters or healthrelated quality of life, individually ${ }^{1,15}$. Furthermore, analyses on sex-differences in psychosocial outcomes are scarce at best in both patients with all-cause or sCeAD related ischemic stroke ${ }^{2,15,16}$. Therefore, our goal was to provide evidence on long-term psychosocial sequelae after sCeAD within our single center cohort study (ReSectstudy) with a special focus on sex differences.

\section{Methods}

Patient recruitment and selection. Details on the ReSect-Study were published previously ${ }^{17,18}$. In short, electronic medical files of patients treated at the Department of Neurology at the Medical University of Innsbruck due to suspected sCeAD between July 1996 and January 2017 were collected and underwent chart review. Subjects were included if intramural hematoma was evident in T1-weighted fat-saturated MRI imaging of cervical vessels and no timely association to high impact trauma existed. All eligible patients were invited to a standardized in-house clinical follow-up visit at least one year after sCeAD event, which included a physical examination as well as psychosocial outcome assessment through questionnaires and detailed history taking by experienced stroke neurologists.

Variable definitions. Clinical and functional status of patients were evaluated using the National Institutes of Health Stroke Scale (NIHSS) at hospital admission and the modified Rankin Scale (mRS) at hospital 


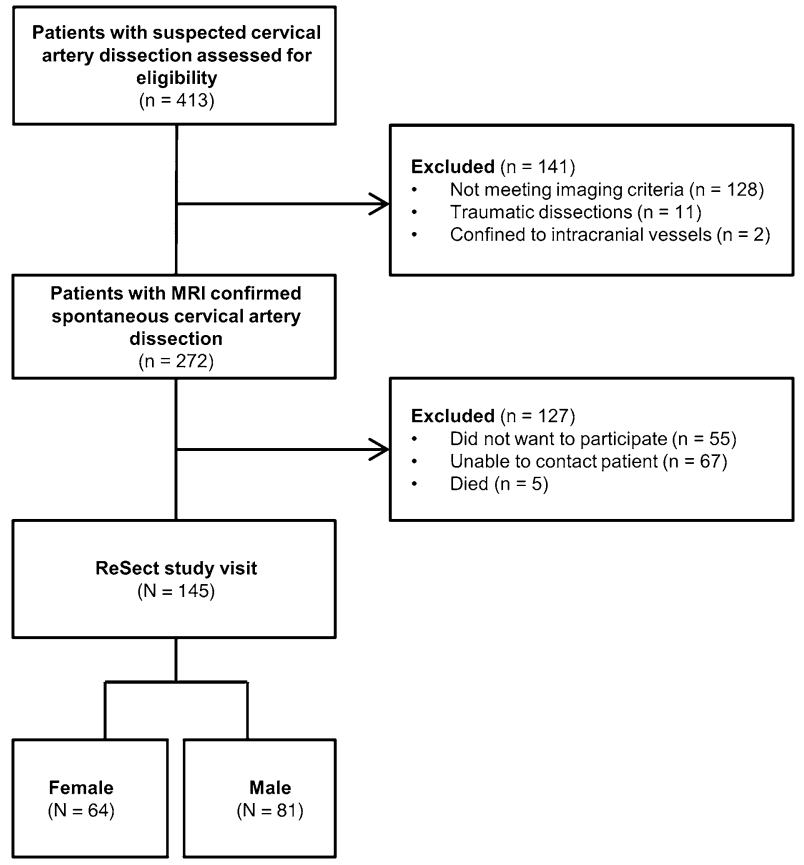

Figure 1. Study flow-chart.

admission,- -discharge and follow-up. Excellent functional outcome was defined as $\mathrm{mRS} \leq 1$. Long-term psychosocial outcome included social reintegration (return to work rate and divorce rate) as well as health related quality of life (HRQoL) measured by WHO Quality of Life-BREF and the 36-Item Short Form Survey (SF 36) questionnaires ${ }^{19,20}$. Return to work was defined as restart of the occupational activity (including stay-at-home parent or caregiver) prior to the index event. The variable was scaled as (1) return to the work in full or reduced capacity (i.e. reduced working hours or in need of assistance for duties previously capable of doing by oneself) or (2) no return to work. The divorce rate was calculated for subjects that were married at baseline.

Statistical methodology. Descriptive statistical analysis was performed using IBM SPSS Statistics (Version 26.0). Differences in categorical variables were examined by Chi-squared test and in continuous variables by the means of Mann-Whitney-U or T-test. A binomial logistic regression model was created to identify independent risk factors for poor functional and social outcome.

Standard protocol approvals, registration, and patient consents. This study was approved by the local ethics committee of the Medical University Innsbruck. All patients that took part in the ReSect-study signed appropriate informed consent. All methods were carried out in accordance with relevant guidelines and regulations.

\section{Results}

In total, $272 \mathrm{sCeAD}$ patients were eligible for inclusion and 145 of these participated in the in-person follow-up evaluation of the ReSect-Study (Fig. 1). The participating subjects did not differ in baseline characteristics to the excluded subjects (Table 1 ).

Five sCeAD patients (1.8\%), three men and two women, died during the survey period between 1996 and 2017. Demographics, baseline characteristics and follow-up parameters of all ReSect subjects are given in Table 1 with sex differences in baseline and follow-up variables depicted in Table 2 . The median age at the initial sCeAD event was 44 (IQR 14.1) years. Of 145 patients, 42 (29\%) presented with local symptoms only (headache, Horner's syndrome, pulsatile tinnitus, cranial nerve palsies). Median time of follow-up was 6.5 years. Excellent functional outcome (mRS $\leq 1)$ was achieved in 129 (89.0\%) patients (Fig. 2).

A total of 127 (87.6\%) sCeAD patients had returned to work and 19 of 108 (17.6\%) married patients had a divorce during follow-up (Fig. 3).

Males predominated in our cohort and were older than women at initial event ( 47.4 vs. 42.6 years; $\mathrm{P}=0.001$ ). Other baseline characteristics, especially measures of physical functioning at baseline (NIHSS, mRS), as well as functional outcome at discharge (mRS $\leq 1$ in men vs. women: $79.0 \%$ vs. $73.4 \% ; \mathrm{P}=0.566)$ and follow-up ( $\mathrm{mRS} \leq 1$ in men vs. women $91.4 \%$ vs. $85.9 \%$; $\mathrm{P}=0.301$ ) did not differ between sexes (Table 2; Fig. 2).

Still, women were less likely to return to work compared to men $(79.7 \%$ vs. $93.8 \% ; \mathrm{P}=0.010)$ and divorce rate after sCeAD was considerably higher in women $(30.2 \%$ vs. $9.2 \% ; \mathrm{P}=0.022)$ (Fig. 3). When building a logistic regression model, the variables with borderline significance between the sexes at baseline $(\mathrm{P} \leq 0.1$ : age, hypertension, NIHSS of 0 at baseline) were independent risk factors for poor functional outcome $(\mathrm{P}=0.001)$ but none of 


\begin{tabular}{|c|c|c|c|}
\hline & ReSect subjects $(n=145)$ & Excluded subjects $(n=127)$ & $P$ value \\
\hline \multicolumn{4}{|l|}{ Baseline } \\
\hline \multicolumn{4}{|l|}{ Demographics } \\
\hline Age $^{\mathrm{b}}$ & $44.3(14.1)$ & $45.2(16.5)$ & $0.75^{\star}$ \\
\hline Male $^{\mathrm{a}}$ & $81(55.9)$ & $82(64.6)$ & $0.14^{\$}$ \\
\hline \multicolumn{4}{|l|}{ Vascular risk factors } \\
\hline Diabetes mellitus $^{\mathrm{a}}$ & $2(1.4)$ & $5(3.9)$ & $0.18^{\$}$ \\
\hline Hypertension $^{\mathrm{a}}$ & $51(35.2)$ & $43(33.9)$ & $0.82^{\$}$ \\
\hline Smoking $^{\mathrm{a}}$ & $43(29.7)$ & $27(25.7)(\mathrm{N}=105)$ & $0.11^{\$}$ \\
\hline Preexisting atherosclerotic disease $\mathrm{e}^{\mathrm{a}}$ & $4(2.8)$ & $2(1.6)$ & $0.51^{\$}$ \\
\hline \multicolumn{4}{|l|}{ sCeAD characteristics } \\
\hline Anterior circulation $^{\mathrm{a}}$ & $67(46.2)$ & $63(49.6)$ & $0.69^{\$}$ \\
\hline Ischemic stroke/TIA ${ }^{\mathrm{a}}$ & $103(71.0)$ & $93(73.2)$ & $0.58^{\S}$ \\
\hline \multicolumn{4}{|l|}{ Stroke characteristics } \\
\hline NIHSS of 0 at baseline ${ }^{\mathrm{a}}$ & $70(49.3)$ & $56(44.4)$ & $0.39^{\$}$ \\
\hline $\mathrm{mRS} \leq 1$ at baseline $\mathrm{e}^{\mathrm{a}}$ & $73(50.3)$ & $56(44.1)$ & $0.16^{\$}$ \\
\hline
\end{tabular}

Table 1. Differences is baseline characteristics of excluded and included subjects. sCeAD, spontaneous cervical artery dissection; TIA, transient ischemic attack. ${ }^{\star}$ Mann-Whitney-U test. ${ }^{\$} \mathrm{Chi}^{2}$-test. ${ }^{\mathrm{a}}$ Variables are given as $\mathrm{n}(\%)$. ${ }^{\mathrm{b}}$ Variables are given as median (interquartile range).

them were independent risk factors for poor social outcome. Solely female sex showed significant association with being divorced upon follow-up ( $\mathrm{P}=0.04$, OR 3.055 [CI 1.03-9.00]). HRQoL and its individual components did not differ significantly between the sexes, even though women in general reported lower values of social and physical functioning after sCeAD (Table 2). HRQoL as well as functional and psychosocial outcomes did not differ in sCeAD patients that presented with stroke / TIA or local symptoms only (data not shown).

\section{Discussion}

Even though sCeAD is one of the lead causes of ischemic stroke in young adults, data on long-term outcome, especially on HRQoL and social reintegration, are scarce. Functional outcome is considered favorable in young ischemic stroke patients in general, which extends to individuals with sCeAD. Still, most analyses to date relied on a rather short follow-up time of usually a couple of months or focused on single outcome parameters ${ }^{8,16,21-26}$. Even though functional outcome is beneficial, patient reported outcomes like reduced HRQoL and suboptimal social reintegration after all-cause young ischemic stroke are also important determinants of successful poststroke recovery on a long run ${ }^{23,27,28}$. To date, little is known about long-term psychosocial sequelae of sCeAD. We present data from a single-center long-term cohort combining functional with psychosocial patient reported outcomes. In general, our study confirms that the beneficial short-term functional outcome after sCeAD persists in the long-run as excellent functional outcome $(\mathrm{mRS} \leq 1)$ was evident in $90 \%$ of patients after a median followup of 6.5 years. Social reintegration parameters overall were in line with the functional metrics as almost $90 \%$ of patients returned to work. This is much higher than the $60 \%$ reported for all-cause young ischemic stroke survivors ${ }^{11-13}$. Furthermore, only one in six subjects had a divorce during the follow-up period. Even though literature to date reports equal outcomes in sexes after $\mathrm{sCeAD}^{1,27,29-33}$, we observed a pronounced gap between men and women with females being significantly less likely to return to work and more likely to get divorced after sCeAD (Fig. 3). Differences between the sexes in psychosocial measures and outcomes have been investigated in detail in other vascular diseases, such as coronary artery disease ${ }^{34-39}$. As psychological factors have a negative impact on outcome of ischemic heart disease, it is tempting to speculate that the same might be true for cerebrovascular diseases, especially in young patients ${ }^{40,41}$.

The little comparison concerning divorce rate that literature offers describes a range from $50 \%$ in rather small case studies of coronary heart disease and a 3 years $7 \%$ divorce rate in all cause young ischemic stroke subjects ${ }^{16,42}$. In both instances, no sex differences had been reported. But, as divorce itself has been linked to an increased risk of acute myocardial infarction, especially in women, the sex differences with women more frequently getting divorced after sCeAD has to be taken seriously ${ }^{43}$. The reasons for the sex differences in our study remain unclear. One might speculate on a gap in psychosocial safety-nets, similar to coronary artery disease patients ${ }^{37}$, or sex specific demands in social functioning. Previous studies have demonstrated that women are more likely to be discharged to assisted living facilities compared to men in general ischemic stroke cohorts ${ }^{44,45}$. Yet, this can be explained by a worse pre-stroke physical functioning in women due to older age at stroke onset and more severe strokes, which was not apparent in our current analysis of sCeAD patients. 


\begin{tabular}{|c|c|c|c|}
\hline & Men & Women & $\mathbf{P}$ \\
\hline \multicolumn{4}{|l|}{ Baseline } \\
\hline \multicolumn{4}{|l|}{ Demographics } \\
\hline Age $^{\mathrm{b}}$ & $47.4(14.2)$ & $42.6(12.7)$ & $0.001^{\star}$ \\
\hline \multicolumn{4}{|l|}{ Vascular risk factors } \\
\hline Diabetes mellitus $^{\mathrm{a}}$ & $2(2.5)$ & $0(0)$ & $0.26^{\$}$ \\
\hline Hypertension $^{\mathrm{a}}$ & $36(44.4)$ & $15(23.4)$ & $0.09^{\$}$ \\
\hline Smoking $^{\mathrm{a}}$ & $21(25.9)$ & $22(34.4)$ & $0.33^{\$}$ \\
\hline Preexisting atherosclerotic disease $\mathrm{a}^{\mathrm{a}}$ & $3(3.7)$ & $1(1.6)$ & $0.42^{\$}$ \\
\hline \multicolumn{4}{|l|}{$s C e A D$ characteristics } \\
\hline Anterior circulation $^{\mathrm{a}}$ & $36(44.4)$ & $31(48.4)$ & $0.63^{\$}$ \\
\hline Ischemic stroke/TIA ${ }^{\mathrm{a}}$ & $58(71.6)$ & $45(70.3)$ & $0.86^{\$}$ \\
\hline \multicolumn{4}{|l|}{ Stroke characteristics } \\
\hline NIHSS of 0 at baseline ${ }^{a}$ & $34(42.0)$ & $37(57.8)$ & $0.10^{\$}$ \\
\hline $\mathrm{mRS} \leq 1$ at baseline $\mathrm{e}^{\mathrm{a}}$ & $40(49.4)$ & $36(56.3)$ & $0.41^{\$}$ \\
\hline \multicolumn{4}{|l|}{ Follow-up } \\
\hline \multicolumn{4}{|l|}{ Functional outcome } \\
\hline mRS 0 at follow-up & $58(71.6)$ & $45(70.3)$ & $0.90^{\$}$ \\
\hline \multicolumn{4}{|l|}{ SF 36} \\
\hline Physical functioning $^{c}$ & $92.5(11.3)$ & $86.3(21.8)$ & $0.05^{-}$ \\
\hline Physical role functioning ${ }^{\mathrm{c}}$ & $92.5(21.4)$ & $87.2(31.5)$ & $0.30^{-}$ \\
\hline Emotional role functioning $^{\mathrm{c}}$ & $91.5(24.7)$ & $89.8(26.5)$ & $0.72^{-}$ \\
\hline Fatigue $^{c}$ & $68.2(13.6)$ & $64.6(14.5)$ & $0.18^{-}$ \\
\hline Emotional well being ${ }^{c}$ & $79.7(13.4)$ & $75.6(17.7)$ & $0.16^{-}$ \\
\hline Social functioning $^{c}$ & $92.3(13.2)$ & $85.7(23.1)$ & $0.06^{-}$ \\
\hline Pain $^{c}$ & $84.3(20.4)$ & $82.2(18.7)$ & $0.57^{-}$ \\
\hline General health $^{c}$ & $75.1(16.5)$ & $72.5(14.9)$ & $0.40^{-}$ \\
\hline \multicolumn{4}{|l|}{ WHO QoL BREF } \\
\hline Physical health $^{c}$ & $86.5(11.8)$ & $84.5(12.5)$ & $0.42^{-}$ \\
\hline Psychological health $^{c}$ & $81.5(11.8)$ & $78.1(16.3)$ & $0.21^{-}$ \\
\hline Social relationships $^{\mathrm{c}}$ & $79.4(16.8)$ & $81.1(15.5)$ & $0.59^{-}$ \\
\hline Environment $^{\mathrm{c}}$ & $87.8(9.4)$ & $87.9(11.7)$ & $0.98^{-}$ \\
\hline
\end{tabular}

Table 2. Differences in baseline and follow-up characteristics between the sexes. sCeAD, spontaneous cervical artery dissection; TIA, transient ischemic attack. ${ }^{\star}$ Mann-Whitney-U Test. ${ }^{\$} \mathrm{Chi}^{2}$-test. ${ }^{-T}$-test. ${ }^{\text {a }}$ Variables are given as $\mathrm{n}(\%) .{ }^{\mathrm{b}}$ Variables are given as median (interquartile range). ${ }^{\mathrm{c}}$ Variables are given as mean (standard deviation).

Strengths of this study are the stringent inclusion criteria, providing a cohort of patients with a definite sCeAD diagnosis. Additionally, data of our well-characterized single-centre cohort rely on long-term standardized in-person follow-up.

Limitations include that not all subjects completed the HRQoL questionnaires. Even though baseline and follow-up characteristics between those assessed within the clinical visits and those evaluated via chart review did not differ (Table 1), we cannot completely rule out a selection bias as patients with low HRQoL and/or depression may be reluctant to participate in a clinical study, as has been described previously on multiple occasions in studies investigating depression ${ }^{46,47}$. Reasons on why subjects did not want to attend the in-house study visit were not routinely assessed during the recruitment phase of the study. Furthermore, we did not record the timing of social reintegration values (divorce and return to work) after sCeAD and had information on divorce proportions but not on other outcomes in long-term unmarried cohabitating relationships. Lastly, it would have been rewarding to present a control group of subjects suffering from other cause ischemic stroke.

In conclusion, even though functional outcome is beneficial in most $\mathrm{sCeAD}$ patients there is a need for measures to prevent poor psychosocial outcome, especially in women. 


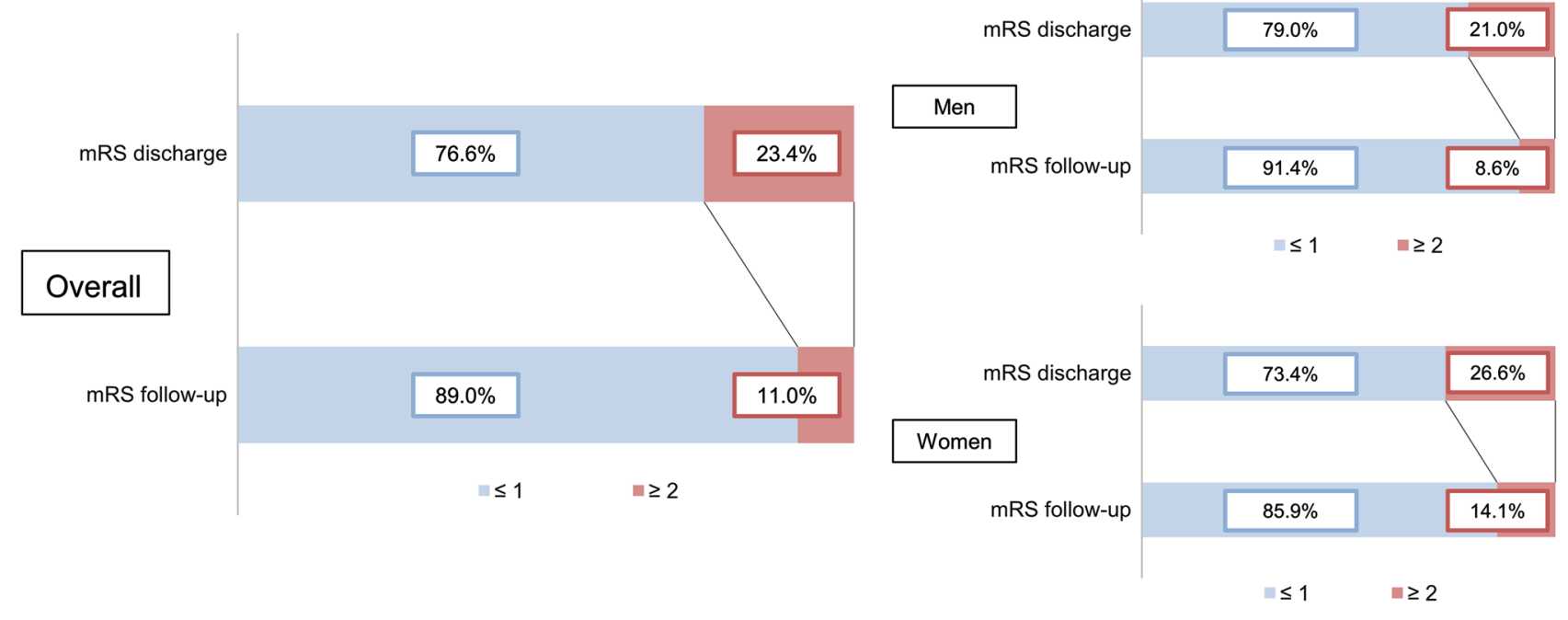

Figure 2. Functional outcome measured by $\mathrm{mRS}$ at discharge and follow-up in all ReSect study participants, as well as men and women separately. Men and women did not differ at discharge $\left(\mathrm{P}=0.566^{\$}\right)$ or follow-up $\left(\mathrm{P}=0.301^{\$}\right)\left({ }^{\$} \mathrm{Chi}^{2}\right.$-test $)$.

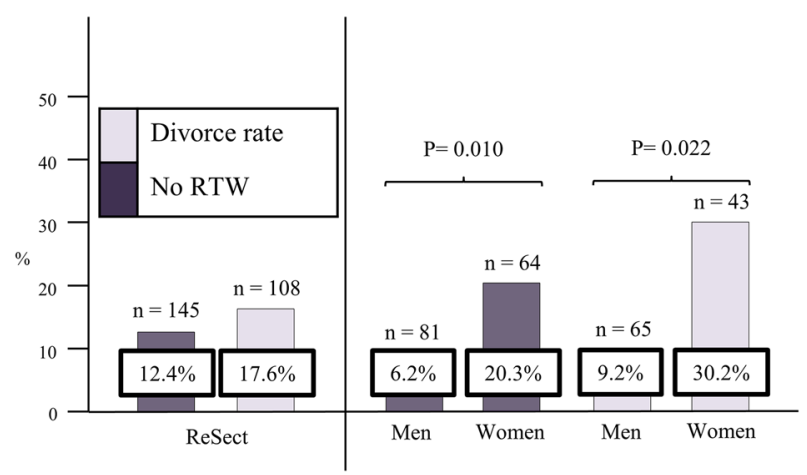

Figure 3. Social reintegration metrics of ReSect-cohort and sex specific subgroup analysis. RTW, return to work.

\section{Data availability}

The datasets generated during and/or analysed during the current study are available from the corresponding author on reasonable request.

Received: 31 August 2021; Accepted: 29 December 2021

Published online: 12 January 2022

\section{References}

1. Arnold, M. et al. Gender differences in spontaneous cervical artery dissection. Neurology 67, 1050-1052 (2006).

2. Czechowsky, D. \& Hill, M. D. Neurological outcome and quality of life after stroke due to vertebral artery dissection. Cerebrovasc. Dis. 13, 192-197 (2002).

3. Touzé, E., Gauvrit, J., Meder, J. \& Mas, J. Prognosis of cervical artery dissection. Front. Neurol. Neurosci. 20, 129-139 (2005).

4. Schievink, W. I. Spontaneous dissection of the carotid and vertebral arteries. N. Engl. J. Med. 344, 898-906 (2001).

5. Fonarow, G. C. et al. Age-related differences in characteristics, performance measures, treatment trends, and outcomes in patients with ischemic stroke. Circulation 121, 879-891 (2010).

6. Knoflach, M. et al. Functional recovery after ischemic stroke-a matter of age: data from the Austrian Stroke Unit Registry. Neurology 78, 279-285 (2012).

7. Waje-Andreassen, U. et al. Ischaemic stroke at a young age is a serious event-final results of a population-based long-term followup in Western Norway. Eur. J. Neurol. 20, 818-823 (2013).

8. Kappelle, L. J. et al. Prognosis of young adults with ischemic stroke: A long-term follow-up study assessing recurrent vascular events and functional outcome in the Iowa Registry of Stroke in Young Adults. Stroke 25, 1360-1365 (1994).

9. Naess, H., Nyland, H. I., Thomassen, L., Aarseth, J. \& Myhr, K.-M. Mild depression in young adults with cerebral infarction at long-term follow-up: a population-based study. Eur. J. Neurol. 12, 194-198 (2005).

10. Persson, J., Ferraz-Nunes, J. \& Karlberg, I. Economic burden of stroke in a large county in Sweden. BMC Health Serv. Res. 12, 341 (2012). 
11. Andersen, G., Christensen, D., Kirkevold, M. \& Johnsen, S. P. Post-stroke fatigue and return to work: A 2-year follow-up. Acta Neurol. Scand. 125, 248-253 (2012).

12. Hofgren, C., Björkdahl, A., Esbjörnsson, E., Sunnerhagen, K. S. \& Stibrant-Sunnerhagen, K. Recovery after stroke: Cognition, ADL function and return to work. Acta Neurol. Scand. 115, 73-80 (2007).

13. Aarnio, K. et al. Return to work after ischemic stroke in young adults. Neurology 91, e1909-e1917 (2018).

14. Gray, L. J. et al. Sex differences in quality of life in stroke survivors: data from the Tinzaparin in Acute Ischaemic Stroke Trial (TAIST). Stroke 38, 2960-2964 (2007).

15. Speck, V., Noble, A., Kollmar, R. \& Schenk, T. Diagnosis of spontaneous cervical artery dissection may be associated with increased prevalence of posttraumatic stress disorder. J. Stroke Cerebrovasc. Dis. 23, 335-342 (2014).

16. Leys, D. et al. Clinical outcome in 287 consecutive young adults (15 to 45 years) with ischemic stroke. Neurology 59, 26-33 (2002).

17. Mayer, L. et al. Local signs and symptoms in spontaneous cervical artery dissection: A single centre cohort study. J. Stroke 21, $112-115$ (2019).

18. Mayer, L. et al. Extracellular matrix protein signature of recurrent spontaneous cervical artery dissection. Neurology 95, e2047e2055 (2020).

19. Skevington, S. M., Lotfy, M. \& O'Connell, K. A. The World Health Organization's WHOQOL-BREF quality of life assessment: Psychometric properties and results of the international field trial-A report from the WHOQOL group. Qual. Life Res. 13, 299-310 (2004).

20. Garratt, A. M., Ruta, D. A., Abdalla, M. I., Buckingham, J. K. \& Russell, I. T. The SF36 health survey questionnaire: An outcome measure suitable for routine use within the NHS?. BMJ 306, 1440-1444 (1993).

21. Lee, V. H., Brown, R. D., Mandrekar, J. N. \& Mokri, B. Incidence and outcome of cervical artery dissection: A population-based study. Neurology 67, 1809-1812 (2006).

22. Béjot, Y., Daubail, B., Debette, S., Durier, J. \& Giroud, M. Incidence and outcome of cerebrovascular events related to cervical artery dissection: The Dijon Stroke Registry. Int. J. Stroke 9, 879-882 (2014).

23. Debette, S. \& Leys, D. Cervical-artery dissections: Predisposing factors, diagnosis, and outcome. Lancet Neurol. 8, 668-678 (2009).

24. de Bruijn, M. A. A. M. et al. Quality of life after young ischemic stroke of mild severity is mainly influenced by psychological factors. J. Stroke Cerebrovasc. Dis. 24, 2183-2188 (2015).

25. Varona, J. F., Bermejo, F., Guerra, J. M. \& Molina, J. A. Long-term prognosis of ischemic stroke in young adults: Study of 272 cases. J. Neurol. 251, 1507-1514 (2004).

26. Goeggel Simonetti, B. et al. Risk factors, aetiology and outcome of ischaemic stroke in young adults: The Swiss Young Stroke Study (SYSS). J. Neurol. 262, 2025-2032 (2015).

27. Fischer, U. et al. Quality of life in survivors after cervical artery dissection. J. Neurol. 256, 443-449 (2009).

28. Naess, H., Waje-Andreassen, U., Thomassen, L., Nyland, H. \& Myhr, K.-M. Health-related quality of life among young adults with ischemic stroke on long-term follow-up. Stroke 37, 1232-1236 (2006).

29. Feigin, V. L. et al. Global burden of stroke and risk factors in 188 countries, during 1990-2013: A systematic analysis for the Global Burden of Disease Study 2013. Lancet Neurol. 15, 913-924 (2016).

30. Maaijwee, N. A. M. M. et al. Long-term increased risk of unemployment after young stroke: A long-term follow-up study. Neurology 83, 1132-1138 (2014).

31. Pozzati, E., Giuliani, G., Acciarri, N. \& Nuzzo, G. Long-term follow-up of occlusive cervical carotid dissection. Stroke 21, 528-531 (1990).

32. Engelter, S. T., Lyrer, P. A., Kirsch, E. C. \& Steck, A. J. Long-term follow-up after extracranial internal carotid artery dissection. Eur. Neurol. 44, 199-204 (2000).

33. Strege, R. J., Kiefer, R. \& Herrmann, M. Contributing factors to quality of life after vertebral artery dissection: A prospective comparative study. BMC Neurol. 19, 312 (2019).

34. Dreyer, R. P. et al. Return to work after acute myocardial infarction: A comparison between young women and men. Circ. Cardiovasc. Qual. Outcomes 9, S45-S52 (2016).

35. Möller-Leimkühler, A. M. Gender differences in cardiovascular disease and comorbid depression. Dialog. Clin. Neurosci. 9, 71-83 (2007).

36. Orth-Gomér, K. et al. Marital stress worsens prognosis in women with coronary heart disease: The Stockholm Female Coronary Risk Study. JAMA 284, 3008-3014 (2000).

37. Leifheit-Limson, E. C. et al. The role of social support in health status and depressive symptoms after acute myocardial infarction: Evidence for a stronger relationship among women. Circ. Cardiovasc. Qual. Outcomes 3, 143-150 (2010).

38. Maas, A. H. E. M. et al. Red alert for women's heart: The urgent need for more research and knowledge on cardiovascular disease in women: proceedings of the workshop held in Brussels on gender differences in cardiovascular disease, 29 September 2010. Eur. Heart J. 32, 1362-1368 (2011).

39. Deter, H.-C. et al. Gender differences in psychosocial outcomes of psychotherapy trial in patients with depression and coronary artery disease. J. Psychosom. Res. 113, 89-99 (2018).

40. Rozanski, A., Blumenthal, J. A., Davidson, K. W., Saab, P. G. \& Kubzansky, L. The epidemiology, pathophysiology, and management of psychosocial risk factors in cardiac practice: The emerging field of behavioral cardiology. J. Am. Coll. Cardiol. 45, 637-651 (2005).

41. Low, C. A., Thurston, R. C. \& Matthews, K. A. Psychosocial factors in the development of heart disease in women: Current research and future directions. Psychosom. Med. 72, 842-854 (2010).

42. Simmonds, R. L., Tylee, A., Walters, P. \& Rose, D. Patients' perceptions of depression and coronary heart disease: A qualitative UPBEAT-UK study. BMC Fam. Pract. 14, 38 (2013).

43. Dupre, M. E., George, L. K., Liu, G. \& Peterson, E. D. Association between divorce and risks for acute myocardial infarction. Circ. Cardiovasc. Qual. Outcomes 8, 244-251 (2015).

44. Petrea, R. E. et al. Gender differences in stroke incidence and poststroke disability in the Framingham heart study. Stroke 40, 1032-1037 (2009).

45. Reeves, M. J. et al. Sex differences in stroke: Epidemiology, clinical presentation, medical care, and outcomes. Lancet Neurol. 7, 915-926 (2008).

46. McDonald, A. M. et al. What influences recruitment to randomised controlled trials? A review of trials funded by two UK funding agencies. Trials 7, 9 (2006).

47. Sully, B. G. O., Julious, S. A. \& Nicholl, J. A reinvestigation of recruitment to randomised, controlled, multicenter trials: A review of trials funded by two UK funding agencies. Trials 14, 166 (2013).

\section{Author contributions}

L.M.-S., M.K. and S.K. conceptualized and designed the study. L.M.-S., M.G., B.D., C.B., L.M.D., S.K. and T.T. were involved in the acquisition of data and data analyses. L.M.-S., M.G. and M.K. interpreted the data. L.M.$\mathrm{S}$. wrote the manuscript. All authors have approved the submitted manuscript version and are accountable for their contributions. 


\section{Funding}

Oesterreichische Nationalbank,15644,15644, Österreichische Forschungsförderungsgesellschaft,16427332,16427332.

\section{Competing interests}

The authors declare no competing interests.

\section{Additional information}

Correspondence and requests for materials should be addressed to L.M.-S.

Reprints and permissions information is available at www.nature.com/reprints.

Publisher's note Springer Nature remains neutral with regard to jurisdictional claims in published maps and institutional affiliations.

(c) (i) Open Access This article is licensed under a Creative Commons Attribution 4.0 International License, which permits use, sharing, adaptation, distribution and reproduction in any medium or format, as long as you give appropriate credit to the original author(s) and the source, provide a link to the Creative Commons licence, and indicate if changes were made. The images or other third party material in this article are included in the article's Creative Commons licence, unless indicated otherwise in a credit line to the material. If material is not included in the article's Creative Commons licence and your intended use is not permitted by statutory regulation or exceeds the permitted use, you will need to obtain permission directly from the copyright holder. To view a copy of this licence, visit http://creativecommons.org/licenses/by/4.0/.

(C) The Author(s) 2022 